\title{
Efeitos do exercício aeróbio durante a hemodiálise em pacientes com doença renal crônica: uma revisão da literatura
}

\author{
Effects of aerobic exercise during haemodialysis in patients with \\ chronic renal disease: a literature review
}

\begin{abstract}
Autores
Joseane Böhm

Mariane Borba Monteiro ${ }^{1}$

Fernando Saldanha Thomé

${ }^{1}$ Centro Universitário Metodista - IPA. ${ }^{2}$ Departamento de Medicina Interna da Universidade Federal do Rio Grande do Sul - UFRGS; Hospital de Clínicas de Porto Alegre - HCPA.
\end{abstract}

Data de submissão: 29/11/2010 Data de aprovação: 06/10/2011

\section{Correspondência para:} Joseane Böhm Avenida do Forte, 113/386 Bairro Vila Ipiranga Porto Alegre - RS - Brasil CEP 91360-000

E-mail: jbohm@pop.com.br

$O$ referido estudo foi realizado no IPA.

Os autores declaram a inexistência de conflitos de interesse.

\section{Resumo}

Introdução: Pacientes com doença renal crônica (DRC) têm capacidade física e funcional reduzida quando comparados à população geral. $\mathrm{O}$ tratamento hemodialítico torna as atividades desses pacientes restritas, favorecendo o estilo de vida sedentário e a limitação funcional. Pacientes em hemodiálise (HD) são menos ativos, apresentam baixa tolerância ao exercício e alto descondicionamento físico. Em virtude dessas alterações, têm sido propostos programas de exercício físico que visam não somente ao tratamento dos sinais clínicos da doença, mas de suas repercussões na função e na qualidade de vida. Objetivos: Realizar uma revisão da literatura sobre os efeitos do exercício físico em pacientes portadores de DRC submetidos a programas de exercício aeróbio em cicloergômetro para membros inferiores durante a HD. Métodos: Foram realizadas consultas às bases de dados Medline, PubMed, Scielo, Embase e ScienceDirect. Resultados: Analisaram-se 14 estudos experimentais randomizados controlados, considerando o período de realização da intervenção na $\mathrm{HD}$, a intensidade e a frequência do exercício, a duração da intervenção e os principais resultados. Conclusão: Os estudos demonstraram que exercícios aeróbios realizados durante a HD promovem a melhora da capacidade aeróbia e o condicionamento físico, além de redução da fadiga e ansiedade, melhora da capilarização muscular e pressão arterial de repouso, aumento no tempo de duração dos exercícios e melhora na depuração da ureia. Palavras-chave: Exercício. Treinamento de resistência. Diálise renal. Consumo de oxigênio. Fadiga.

\section{Abstract}

Introduction: Patients with chronic kidney disease (CKD) have reduced physical and functional capacity when compared with the general population. Hemodialysis (HD) restricts patients activities, favoring a sedentary lifestyle and leading to functional limitations. HD patients are less active, present low exercise tolerance and have high physical deconditioning. Physical exercise programs have been proposed as a strategy not only to treat clinical symptoms, but also to reduce physical limitations and improve the quality of life of these patients. Objectives: The purpose of this study was to review the literature on the effects of physical exercise (aerobic exercise on a cycle ergometer for the lower limbs) on CKD undergoing HD. Methods: The Medline, PubMed, Scielo, Embase and DirectScience databases were searched. Results: Fourteen randomized controlled clinical trials were analyzed as regards the timing of the intervention during the HD session, exercise intensity and frequency, the duration of the intervention and the main results. Conclusion: The studies demonstrated that aerobic exercises performed during HD improve the aerobic capacity and physical conditioning, reduce fatigue and anxiety, improve muscle capillarization and resting blood pressure, increase exercise duration and improve urea clearance.

Keywords: Exercise. Resistance training. Renal dialysis. Oxygen uptake. Fatigue. 


\section{INTRODUÇÃO}

A doença renal crônica (DRC) é a perda lenta, progressiva e irreversível da função renal, na qual o organismo não mantém o equilíbrio metabólico e hidroeletrolítico. É considerado um grande problema de saúde pública por suas elevadas taxas de morbidade $\mathrm{e}$ mortalidade e tem impacto negativo sobre a qualidade de vida relacionada à saúde. ${ }^{1}$

Dados apresentados em janeiro de 2009 pela Sociedade Brasileira de Nefrologia ${ }^{2}$ (SBN) demonstram que, aproximadamente, 77.589 pacientes realizaram tratamento dialítico e $89,6 \%$ destes encontravam-se em hemodiálise (HD). A prevalência correspondeu a 405 pacientes em HD por milhão de habitantes e $57,3 \%$ possuíam idade entre $18-60$ anos. A taxa de mortalidade foi de $17,1 \%$ e o gasto com programas de diálise e transplante renal no Brasil situou-se ao redor de 1,4 bilhões de reais ao ano. ${ }^{3}$

Pacientes com DRC apresentam menor capacidade física e funcional quando comparados à população geral ${ }^{4}$ e o tratamento de HD torna as atividades desses pacientes limitadas após o início do tratamento, favorecendo o sedentarismo e a limitação funcional. ${ }^{5}$ Pacientes em HD são menos ativos, apresentam baixa tolerância ao exercício e alto descondicionamento físico, provavelmente relacionados à atrofia muscular, anemia, miopatia e neuropatia urêmica, disfunção autonômica, diminuição da flexibilidade, redução da força muscular, má nutrição e comorbidades associadas. ${ }^{6}$

Em virtude dessas alterações, têm sido propostos programas de exercício físico que visam não somente ao tratamento dos sinais clínicos da doença, mas à sua repercussão nas funções cardiorrespiratória e muscular, o que proporciona melhora na qualidade de vida. ${ }^{7,8}$

O objetivo deste estudo foi realizar uma revisão da literatura sobre os efeitos do exercício físico em pacientes portadores de DRC submetidos a programas de exercício aeróbio em cicloergômetro para membros inferiores durante a HD.

\section{Métodos}

Para a realização deste estudo, foram consultadas as bases de dados Medline, PubMed, Scielo, Embase e ScienceDirect, em julho de 2010, utilizando os seguintes critérios prévios: data de publicação entre janeiro de 2002 e julho de 2010; idiomas, português e inglês; descritores incluídos no título e/ou resumo - exercício aeróbio (aerobic exercise), hemodiálise (haemodialysis) e doença renal crônica (chronic kidney disease).
Para a seleção dos artigos, inicialmente, foi feita a leitura dos resumos, verificando se continham as informações que preenchiam os seguintes critérios de inclusão: estudos com participantes adultos maiores de dezoito anos, portadores de DRC em tratamento de HD por mais de três meses e estudos experimentais randomizados controlados, que avaliaram os efeitos do exercício aeróbio realizado em cicloergômetro para membros inferiores durante a HD.

Os estudos que, além de participantes em tratamento de HD, também envolviam grupos em tratamento de HD peritoneal, indivíduos saudáveis sedentários e indivíduos em exercício domiciliar, foram incluídos, porém, somente os resultados relacionados aos participantes em HD foram considerados. Os artigos da seleção final foram lidos na íntegra.

\section{Resultados}

Foram analisados quatorze estudos experimentais randomizados controlados, considerando o período de realização do exercício durante a HD, a intensidade e a frequência do exercício, a duração do estudo e os principais resultados.

O período de realização do exercício ocorreu, em média, na segunda hora de HD. Quatro estudos ${ }^{9-12}$ não informaram o período de realização do exercício, um realizou a intervenção durante a primeira hora $^{13}$ e outro utilizou os primeiros noventa minutos de HD. ${ }^{14} \mathrm{~A}$ intensidade do exercício variou entre 40-60\% do pico de consumo máximo de oxigênio $\left(\mathrm{VO}_{2 \max }\right)^{14-16}$ e entre $50-85 \%$ da frequência cardíaca máxima $\left(\mathrm{FC}_{\text {мах }}\right)$ de treinamento. ${ }^{9,10,12,13,17,18}$ Dois estudos ${ }^{4,11}$ utilizaram o percentual de limiar anaeróbio e outros sete ${ }^{10,12,15,16,19-21}$ utilizaram a Escala de Percepção de Esforço de Borg para a prescrição do exercício. A frequência semanal utilizada pela maioria dos estudos foi de três vezes por semana. Dois estudos $^{12,15}$ utilizaram duas sessões de exercícios por semana. A duração total dos protocolos de exercícios físicos descritos nos artigos variou entre 6 e 40 semanas. A Tabela 1 mostra as principais características e resultados dos estudos analisados.

\section{Dıscussão}

Diversos estudos demonstram o prejuízo cardiorrespiratório de pacientes com DRC. O pico de $\mathrm{VO}_{2 \text { máx }}$ desses pacientes corresponde a $15-21 \mathrm{~mL} / \mathrm{kg} / \mathrm{min},{ }^{1}$ níveis que são 20 - 50\% mais baixos do que valores encontrados em sujeitos saudáveis sedentários, ${ }^{22}$ nos quais estes valores variam entre 35 e $40 \mathrm{~mL} / \mathrm{kg} / \mathrm{min} .{ }^{1}$ A realização de exercícios aeróbios em pacientes em 


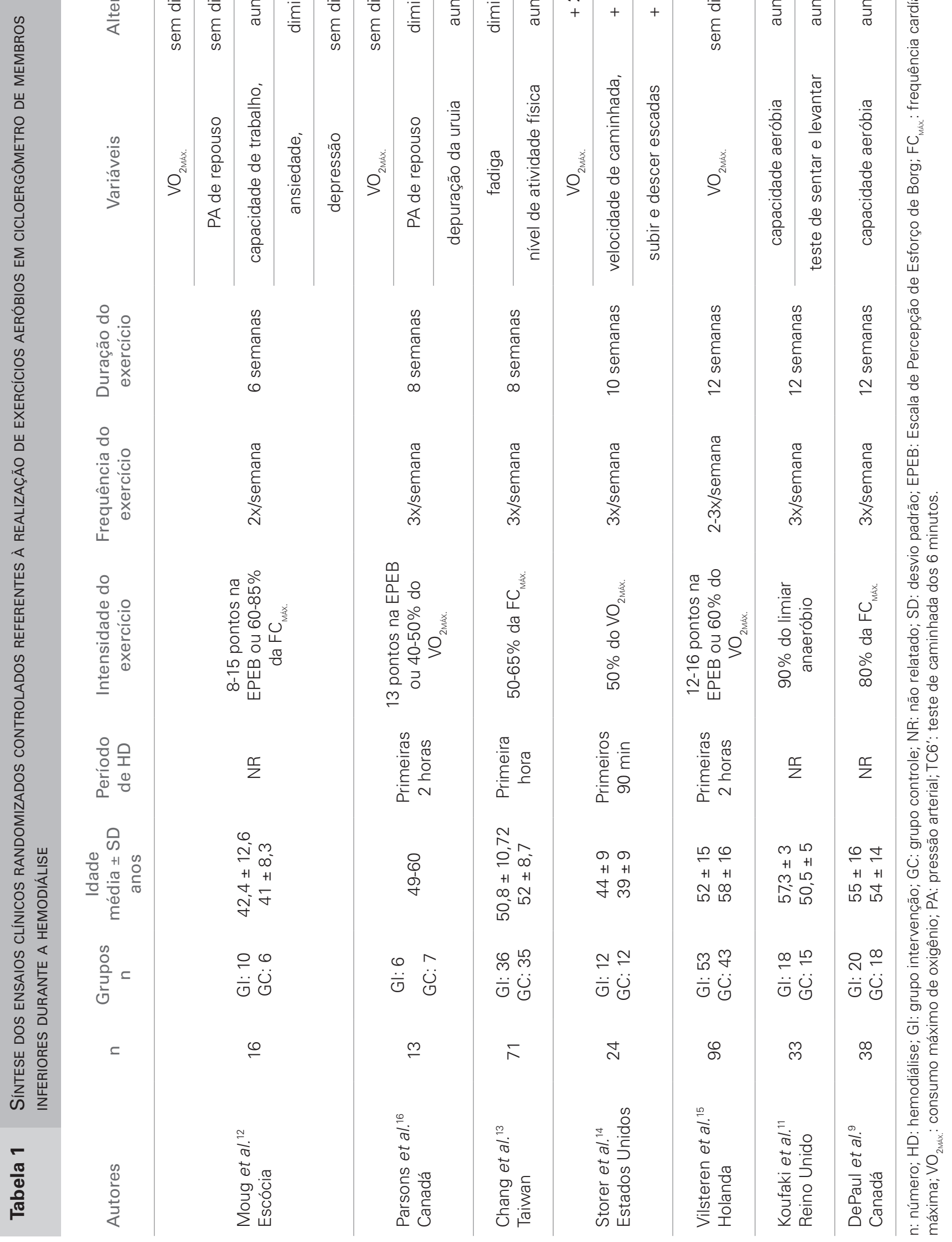




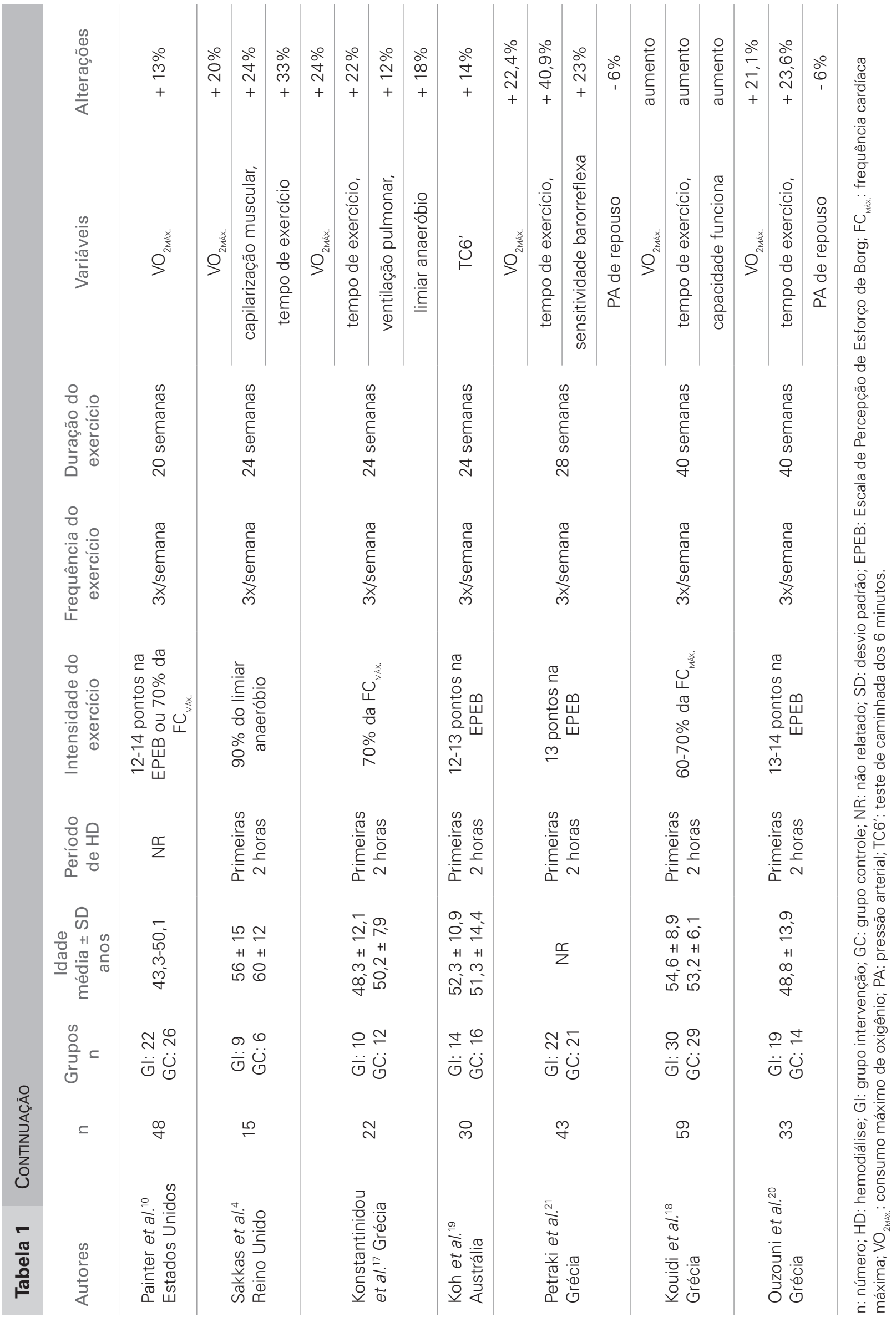


HD tem mostrado efeitos benéficos na melhora da capacidade cardiorrespiratória.

Avaliando os efeitos do treinamento aeróbio com intensidade de $50 \%$ do $\mathrm{VO}_{2 \text { máx }}$, Storer et al. ${ }^{14}$ demonstraram que houve melhora no desempenho físico do grupo intervenção, mesmo com a baixa intensidade de exercício aeróbio realizado em cicloergômetro de membros inferiores. Apesar da melhora apresentada pelos participantes que realizaram o exercício, o $\mathrm{VO}_{2 \text { máx }}$ permaneceu $30 \%$ abaixo do obtido no grupo de indivíduos saudáveis. Os autores sugerem que o exercício aeróbio de baixa intensidade não é suficiente para restabelecer a capacidade funcional igual à de indivíduos saudáveis devido à presença de miopatia urêmica e outras patologias associadas.

Aumentos entre $13-40 \%$ no $\mathrm{VO}_{2 \text { máx }}$ têm sido relatados depois de treinamentos com duração de 8 a 40 semanas..$^{4,10,14,17,18,20,21}$ Painter et al. ${ }^{10}$ observaram aumento de $13 \%$ no pico de $\mathrm{VO}_{2 \text { máx }}$ após vinte semanas de treinamento. Paralelamente, não observaram relação entre o aumento do consumo de oxigênio e o nível de hematócrito.

DePaul et al. ${ }^{9}$ verificaram melhora na capacidade aeróbia no grupo intervenção após 12 semanas de treinamento, porém, essa melhora não foi sustentada 5 meses após o término do treinamento, indicando a necessidade de continuidade na realização de exercícios. Os resultados do teste de caminhada de seis minutos não mostraram diferenças estatisticamente significativas, dados também relatados por Koh et al. ${ }^{19}$ após 24 semanas de treinamento aeróbio.

Avaliando os efeitos do treinamento aeróbio, utilizando $90 \%$ do limiar anaeróbio para a prescrição do exercício, Sakkas et al. ${ }^{4}$ observaram aumento de $24 \%$ na capilarização muscular, $33 \%$ no tempo de duração do exercício e $20 \%$ no pico de $\mathrm{VO}_{2}$ máx. Os autores relatam que a insuficiência renal não danifica a cadeia respiratória enzimática e o sistema musculoesquelético de pacientes urêmicos responde ao estímulo do exercício da mesma forma que a população em geral. Utilizando a mesma intensidade de treinamento, Koufaki et al. ${ }^{11}$ encontraram melhora da capacidade aeróbia dos pacientes que realizaram a intervenção, com diminuição no tempo de execução do teste de sentar e levantar, enquanto os pacientes do grupo controle apresentaram diminuição da capacidade aeróbia nas doze semanas do estudo.

Konstantinidou et al. ${ }^{17}$ compararam os efeitos de três modelos de treinamento: em dias de não $\mathrm{HD}$, durante a HD e domiciliar. A capacidade aeróbia melhorou nos três modelos de treinamento e os resultados foram mais evidentes no treinamento em dias de não HD. Apesar disso, quando inquiridos, os pacientes responderam que preferiam realizar os exercícios durante a sessão de HD.

Após um programa de reabilitação de 40 semanas, Kouidi et al. ${ }^{18}$ relataram que a melhora na capacidade aeróbia está associada a alterações na capacidade funcional do ventrículo esquerdo, melhorando alguns indicadores de risco de morte súbita em pacientes em HD. É recomendado que o exercício aeróbio seja realizado nas primeiras duas horas de HD, pois, após esse período, podem ocorrer instabilidades cardiovasculares que impedem a realização de exercícios. ${ }^{1}$

Ouzouni et al. ${ }^{20}$ também utilizaram um período de estudo de quarenta semanas e verificaram aumento de $21,1 \%$ no pico de $\mathrm{VO}_{2 \text { máx }}$, melhora de $23,6 \%$ no tempo de duração do exercício e diminuição de $6 \%$ da pressão arterial (PA) de repouso. No estudo de Petraki et al. ${ }^{21}$ foi observado aumento de $22,4 \%$ no pico de $\mathrm{VO}_{2 \text { máx }}$, melhora de $40,9 \%$ no tempo de duração do exercício e $23 \%$ no índice de sensitividade barorreflexa arterial, com diminuição de $6 \%$ da PA de repouso, indicando melhora no índice de controle autonômico cardíaco.

Não foram observadas alterações na capacidade máxima de trabalho nos pacientes avaliados por Parsons et al., ${ }^{16}$ mas esses autores demonstraram que, com a realização do exercício aeróbio, houve diminuição na PA de repouso e melhora na eficácia da depuração da ureia durante a HD. Moug et al. ${ }^{12}$ não encontraram alterações significativas na capacidade aeróbia e na PA de repouso, apesar da melhora na capacidade de trabalho e na diminuição da ansiedade.

Van Vilsteren et al. ${ }^{15}$ não relataram alterações significativas no pico de $\mathrm{VO}_{2 \text { máx }}$ realizando exercício aeróbio com baixa e moderada intensidade devido à fadiga muscular dos pacientes gerada após a realização de exercícios de força no período pré-dialítico, mas os autores observaram que a presença de um supervisor durante os exercícios oferece suporte motivacional e estímulo para que os pacientes mantenham-se ativos e aumentem a adesão ao programa de treinamento.

Em contrapartida, Chang et al. ${ }^{13}$ observaram que pacientes ativos demonstram menos fadiga e melhora no nível de atividade física entre a quarta e a oitava semana de treinamento. Os pacientes do grupo intervenção apresentaram diminuição contínua nos níveis de fadiga e a melhora no condicionamento foi evidente em pacientes que já realizavam exercícios físicos. Os autores concluem que a realização de exercícios durante a sessão de HD é seguro, não oferece custos, nem há a necessidade de disponibilizar tempo extra 
pelo paciente, podendo ser considerada um elemento rotineiro durante a realização da $\mathrm{HD}$.

\section{Conclusão}

Após a revisão da literatura, pode-se concluir que a realização de exercícios aeróbios durante a HD melhora a capacidade funcional, proporcionando benefícios cardiorrespiratórios e musculares aos pacientes. Os estudos demonstraram que os exercícios promovem a melhora da capacidade aeróbia e o condicionamento físico, diminuição da fadiga e da ansiedade, melhora da capilarização muscular e da PA de repouso, aumento no tempo de duração dos exercícios e melhora na depuração da ureia.

Há diversidade quanto à forma de aplicação desses programas em termos de frequência, intensidade e duração, devendo o programa proposto ser adequado à realidade de cada serviço e de cada paciente. Uma vez que a DRC pode ter influência significativa na qualidade de vida, torna-se fundamental a realização de estudos que ofereçam alternativas de tratamento que promovam melhora no quadro clínico, diminuindo a presença de comorbidades e os índices de mortalidade.

A presença de um supervisor durante a realização dos exercícios oferece suporte motivacional e estímulo para que os pacientes se mantenham ativos, aumentando a adesão ao programa de treinamento. A realização de exercícios aeróbios deve ser contínua, pois é segura, não oferece custos ao paciente, nem há a necessidade de disponibilizar tempo extra, podendo ser considerada um elemento rotineiro durante a realização da HD.

\section{REFERÊNCIAS}

1. Soares A, Zehetmeyer M, Rabuske M. Atuação da fisioterapia durante a hemodiálise visando à qualidade de vida do paciente renal crônico. Rev Saúde da UCPEL 2007;1:7-12.

2. Sesso RCC. Censo brasileiro de diálise 2009. [Internet]. [cited 2010 Sep]. Available from: http://www.sbn.org.br

3. Romão Júnior JE. Doença renal crônica: definição, epidemiologia e classificação. J Bras Nefrol 2004;26:1-3.

4. Sakkas GK, Sargeant AJ, Mercer TH, et al. Changes in muscle morphology in dialysis patients after 6 months of aerobic exercise training. Nephrol Dial Transplant 2003;18:1854-61.

5. Martins IRA, Cesarino BC. Qualidade de vida de pessoas com doença renal crônica em tratamento hemodialítico. Rev Lat Am Enfermagem 2005;13:670-6.

6. Coelho MD, Castro AM, Tavares HA, et al. Efeitos de um programa de exercícios físicos no condicionamento de pacientes em hemodiálise. J Bras Nefrol 1998;20:207-10.

7. Daul AE, Schafers RF, Daul K, et al. Exercise during hemodialysis. Clin Nephrol 2004;61:26-30.

8. Martins MRI, Cesarino CB. Atualização sobre programas de educação e reabilitação para pacientes renais crônicos submetidos à hemodiálise. J Bras Nefrol 2004;26:45-50.

9. DePaul V, Moreland J, Eager T, et al. The effectiveness of aerobic and muscle strength training in patients receiving haemodialysis and EPO: a randomized controlled trial. Am J Kidney Dis 2002;40:1219-29.

10. Painter P, Moore G, Carlson L, et al. Effects on exercise training plus normalization of hematocrit on exercise capacity and health-related quality of life. Am J Kidney Dis 2002;39:257-65.

11. Koufaki P, Mercer TH, Naish PF. Effects of exercise training on aerobic and functional capacity of endstage renal disease patients. Clin Physiol Funct Imaging 2002;22:115-24.

12. Moug SJ, Grant S, Creed G, et al. Exercise during haemodialysis: west of Scotland pilot study. Scott Med J 2004;49:14-7.

13. Chang Y, Cheng S, Lin M, et al. The effectiveness of intradialytic leg ergometry exercise for improving sedentary life style and fatigue among patients with chronic kidney disease: a randomized clinical trial. Int J Nurs Stud 2010;47:1383-8.

14. Storer TW, Casaburi R, Sawelson S, et al. Endurance exercise training during haemodialysis improves strength, power, fatigability and physical performance in maintenance haemodialysis patients. Nephrol Dial Transplant 2005;20:1429-37.

15. Vilsteren MCBA, Greef MHG, Huisman RM. The effects of a low-to-moderate intensity pre-conditioning exercise programme linked with exercise counselling for sedentary haemodialysis patients in The Netherlands: results of a randomized clinical trial. Nephrol Dial Transplant 2005;20:141-6.

16. Parsons TL, Toffelmire EB, King-VanVlack CE. The effect of an exercise program during hemodialysis on dialysis efficacy, blood pressure and quality of life in end-stage renal disease (ESRD) patients. Clin Nephrol 2004;61:261-74.

17. Konstantinidou E, Koukouvou G, Kouidi E, et al. Exercise training in patients with end-stage renal disease on haemodialysis: comparison of three rehabilitation programs. J Rehabil Med 2002;34:40-5.

18. Kouidi EJ, Grekas DM, Deligiannis AP. Effects of exercise training on noninvasive cardiac measures in patients undergoing long-term hemodialysis: a randomized controlled trial. Am J Kidney Dis 2009;54:511-21.

19. Koh KP, Fassett RG, Sharman JE, et al. Effect of intradialytic versus home-based aerobic exercise training on physical function and vascular parameters in haemodialysis patients: a randomized pilot study. Am J Kidney Dis 2010;55:88-99.

20. Ouzouni S, Kouidi E, Sioulis A, et al. Effects of intradialytic exercise training on health-related quality of life indices in haemodialysis patients. Clin Rehabil Med 2009;23:53-63.

21. Petraki M, Kouidi E, Grekas D, et al. Effects of exercise training during haemodialysis on cardiac barorreflex sensitivity. Clin Nephrol 2008;70:210-9.

22. Bohm CJ, Ho J, Duhamel TA. Regular physical activity and exercise therapy in end-stage renal disease: how should we "move" forward? J Nephrol 2010;23:235-43. 